\title{
Experimental Investigation of Reversible Modulation of Biological Fluid Crystallogenesis
}

\author{
Andrew K. Martusevich1,2, Alexander G. Samodelkin1, Lida K. Kovaleva3 \\ ${ }^{1}$ Nizhny Novgorod State Agricultural Academy, Nizhny Novgorod, Russia \\ ${ }^{2}$ Privolzhsky Federal Medical Research Centre, Nizhny Novgorod, Russia \\ ${ }^{3}$ Kirov State Medical Academy, Kirov, Russia \\ Email: cryst-mart@yandex.ru
}

Received 3 December 2015; accepted 17 January 2016; published 20 January 2016

Copyright (C) 2016 by authors and Scientific Research Publishing Inc.

This work is licensed under the Creative Commons Attribution International License (CC BY).

http://creativecommons.org/licenses/by/4.0/

(c) (i) Open Access

\begin{abstract}
Free and initiated by $0.9 \%$ sodium chloride solution yielding of crystals was studied in the urine of the 35 healthy people. The metabolites-sodium lactate and sodium pyruvate-were superinduced into the dryable biological system in the increasing physiological concentrations. Regularities of the neogenic mixtures' crystallization were revealed according to the metabolites dose. We showed the antagonistic tendencies in the parameters' change at the increasing concentrations of the different metabolites-substratum of the enzyme's opposite reactions-relying on the comparative estimation of the crystalloscopical and tezigraphical indices' dynamics. Our own data and literature information brought us to a new conception of the biofluid's stability as a crystallogenetic solution. According to it any substance injected into a dehydrated biological system proves to be a stabilizing or destabilizing agent which leads to a significant reorganization of the biocrystallogenesis. This phenomenon may cause a development of a management strategy of the biological crystallogenesis processes.
\end{abstract}

\section{Keywords}

Crystallogenesis, Urine, Sodium Lactate, Sodium Pyruvate

\section{Introduction}

Today a new scientific field based on application of the crystalloscopic analysis of the biological substances develops actively [1]-[3]. We call this biomedical discipline biocrystallomics. This special term is necessary for its differentiation from "classic" crystallography, which has a methodology and methods for estimation of physical 
and chemical properties of different crystal matters. At sane time, "classic" crystallography could not study the diagnostic information of dehydrated specimens of complex heterogeneous solutions, especially biological substrates.

The most researches' tasks on this theme include only revelation of the diagnostic sense of the biological substrate crystallogenesis' phenomenon by looking — for the specific "marker" structures [4] [5], or to a smaller extent evaluative criterions [6]-[9]. Some authors have studied the role of the crystalloscopic methods in the estimation of the treatment effectiveness [10]-[12]. There are also solitary facts on experimental biocrystallomics, i.e. on examination of the biological mediums and their components "behavior" under crystallization depending on the different types of modulators-physical, chemical, biological. For example, a character of the simulated protein and protein-saline solutions crystallization is described [13] [14]. An individual crystallogenesis of some organic and non-organic acids and salts is estimated [15]. But it should be mentioned that these works touch slightly upon the question about the character of the native biological mediums crystallization [16].

It is very interesting to model physiological and pathological states concerned with the increase and decrease of some metabolites concentration in the biological fluid, first of all those which are antagonists to liquid biological systems. In such a case it is suitable to use the direct and back reactions substratum of the biosubstances enzyme complexes.

That is why the aim of this research was to study the biofluids crystallization reformed character under administration of the biochemically opposite substances by the urine example.

\section{Materials and Methods}

\subsection{Material}

We used 35 specimens of the human urine as a basic biological fluid. Urine samples were collected from healthy volunteers. Our study was approved with Local Ethic Committee of Nizhny Novgorod State Agricultural Academy (Nizhny Novgorod, Russia). All volunteers signed an informed consent for participation in this study.

\subsection{Protocol of Exposure on Urine Samples}

Aqueous solutions of sodium lactate and sodium pyruvate were added to the biological medium. We used $40 \%$, $20 \%, 10 \%, 5 \%$ and $2.5 \%$ concentrations of sodium lactate and 200, 100, 50, 25 and $12.5 \mathrm{mg} / \mathrm{ml}$ concentrations of sodium pyruvate. Minimal concentration of both substances corresponds to its physiological values [17] [18]. That is why we approximated the model hyperlactateuria and hyperpyruvateuria to theoretically possible for an individual under pathology conditions.

\subsection{Method of Crystallization Study}

Free and initiated by $0.9 \%$ sodium chloride solution crystallogenesis of the formed biosystems was studied. We used the original criterions to estimate crystalloscopic and tezigraphic facias [19] [20]. As the main parameters structure index (SI), crystallizability (Cr) and facia's destruction degree (FDD) were used to describe free crystallogenesis, and main tezigraphic coefficient $(\mathrm{Q})$, belt coefficient $(\mathrm{P})$ and FDD were used for the comparative tezigraphy data [21]. These indices have the same information sense at crystalloscopic and tezigraphic specimens deciphering. All parameters for crystalloscopic facia (SI, Cr and FDD) were estimated in straight point scale with a minimum corresponding to 0 points and a maximum corresponding to 3 points. Calculation of FDD for tezigraphic facias was similar to crystalloscopic one. Main tezigraphic coefficient (Q) was calculated a as the number of crystallization centers in the sample, including biological fluid and $0.9 \%$ sodium chloride solution, and the sample obtained only from sodium chloride. Belt coefficient $(\mathrm{P})$ is the ratio of the maximum and minimum diameters of the belts formed in teziographic facia.

\subsection{Statistical Analysis}

Statistical analysis of the data was performed with Statistica 6.0 program. Data were expressed as means \pm SE, the Student's t-test was used for detection of statistical difference.

\section{Results and Discussion}

Free and initiated crystallogenesis analysis showed a distinct transformation dynamics of both crystallogenesis 
types according to the input metabolites concentrations (Figure 1 and Figure 2).

Particularly we revealed considerable changes in the crystallogenesis of the neogenic complex solutions"urine-sodium lactate" and "urine-sodium pyruvate", in conformity with the most significant criterions which qualitatively and quantitatively characterized biosystem's capacity for yielding of crystal. So increase of the sodium lactate concentration in the absence of active crystals former (according to the crystalloscopic test) resulted in a regular structure transgression and picture chaotic state. The structure index dynamics was evidence of this (Figure 1). At the same time rise of the sodium lactate concentration showed an opposite tendency. Besides administration of the sodium pyruvate increasing concentrations (which had ability for a polymerization and consequently gelatinization according to the chemical composition) complicated the crystallization centers forming. This was showed by the crystallizability level changes. Even formed crystalline structures had a high destruction degree. Sodium lactate had polysemantic tendencies.

It was determined however that the addition to the containing lactate dehydrogenase biosystem substratum of its direct and back reactions in the increasing concentrations resulted in the opposite dynamics of the indices (according to the comparative analysis of the Figure 1). Such a regularity existed independently of the curve complexity which represented the parameters dynamics. This fact showed that the enzyme activation determined transformation of the biofluid's ability for yielding of crystal (on conditions that the biofluid included the enzyme). This perhaps was caused by the conformational transformations of the enzyme as a big protein.
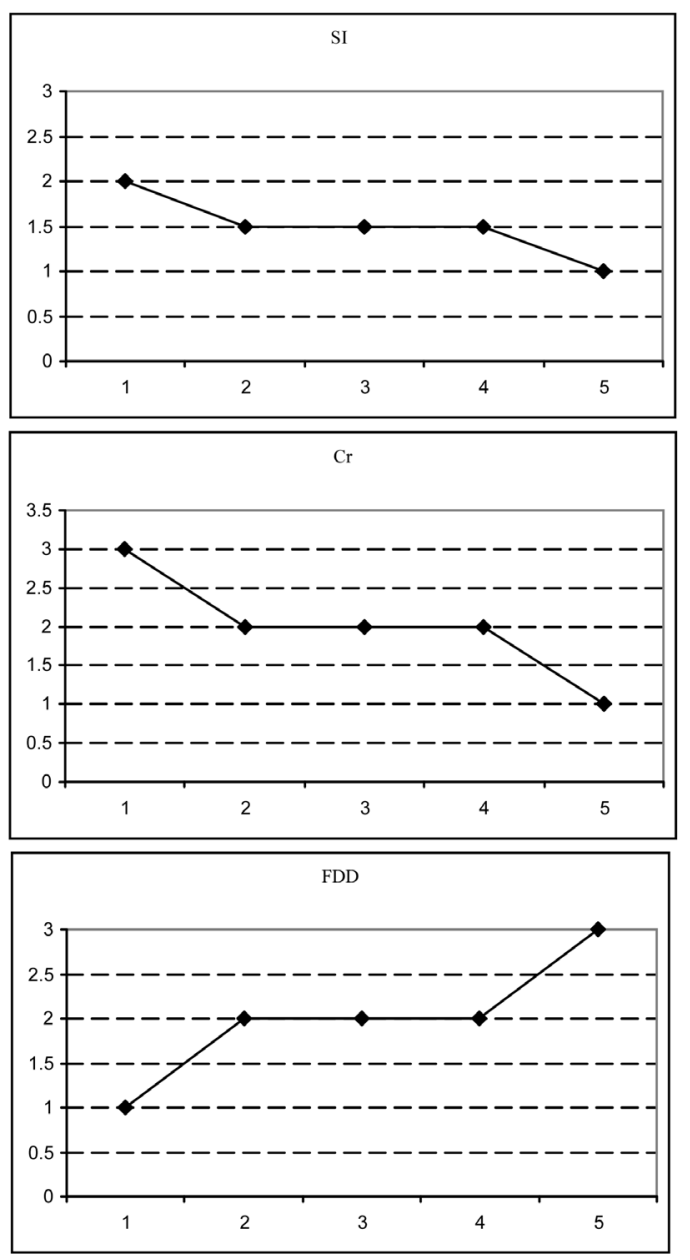

(a)

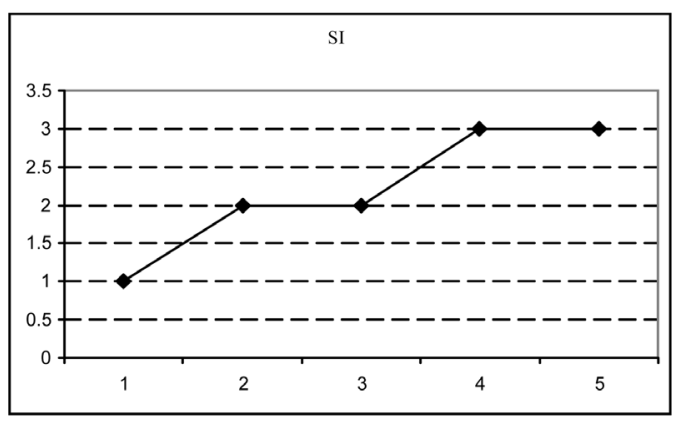

$\mathrm{Cr}$
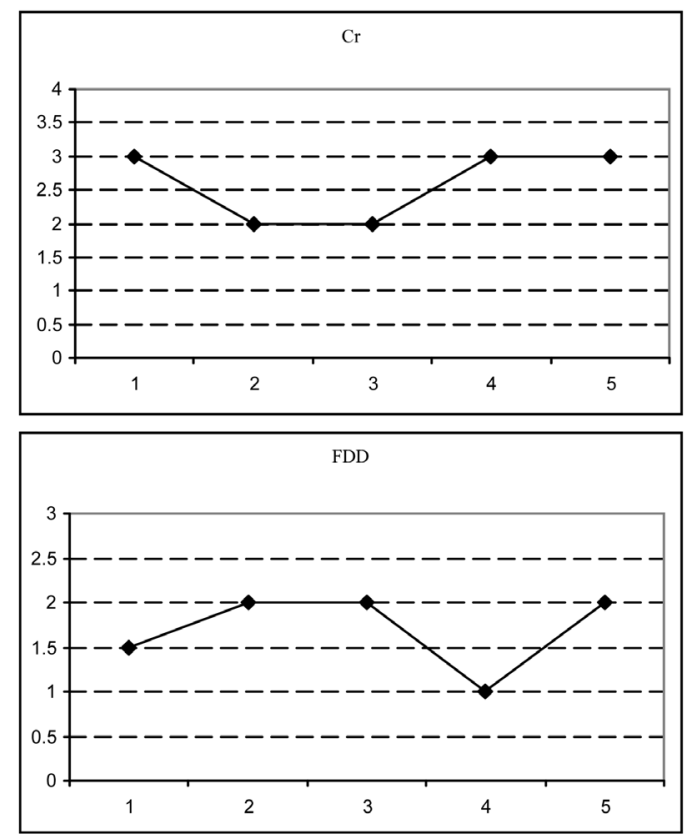

(b)

Figure 1. Transformation of the urine own crystallogenesis under the metabolites addition (all data are shown in OY axis indicated in points; mean values of all parameters are illustrated). (a) Administration of sodium lactate into the biofluid; (b) Administration of sodium pyruvate into the biofluid. SI—structure index; $\mathrm{Cr}$-crystallizability; FDD—phase's destruction degree. 

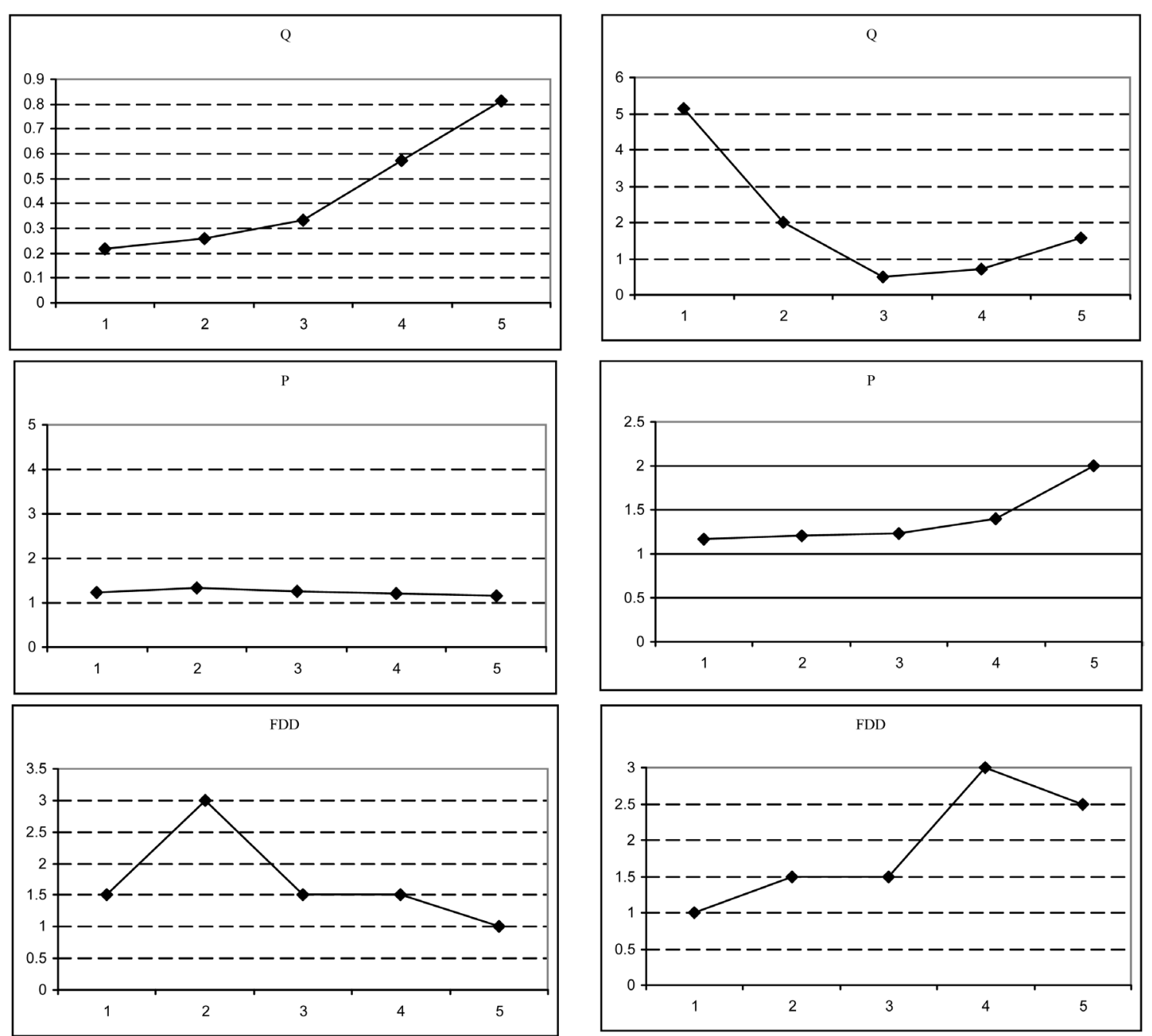

(a)

(b)

Figure 2 . The initiation of the $0.9 \%$ sodium chloride solution crystallization by urine under the metabolites addition (all data are shown in OY axis indicated in points; mean values of all parameters are illustrated). (a) Administration of sodium lactate into the biofluid; (b) Administration of sodium pyruvate into the biofluid. Q — main tezigraphic coefficient; P-belt coefficient; FDD—phase's destruction degree.

It was ascertained by the analysis of the biosystem's crystallogenesis results initiated by the $0.9 \%$ sodium chloride solution that the increase of the sodium lactate concentration brought to the initiative potential intensification towards the basic biological fluid (according to the most significant quantitative index of the tezigraphic test-main tezigraphic coefficient Q). Sodium pyruvate however reduced the biofluid's initiative properties pro rata its concentration (Figure 2).

The belt coefficient (P) and FDD dynamics had not a regular character because the modifications made with the model biofluid change not the protein composition of the neogenic biological system.

But even a complicated metabolites concentration dependence of the specimen destruction degree (and the main tezigraphic coefficient) was not accidental when the analysis was made in the comparative aspect. In this case the biosubstratum concentration increase of the one enzyme opposite reactions led to the parameters antagonistic changes (according to the data of the biosystem's own crystallization) (Figure 2).

From the dehydration (of the crystalloscopic or tezigraphic phase) terminal result point of view we divided logically all the factors into stabilizing (made for keeping of the biosystem as a fluid) and destabilizing (which caused a phase change to a solid) concerning the biofluid's physical state. Main factors of each above-listed groups are shown on the Figure 3. If the destabilizing factors prevail at the dehydration we see: 


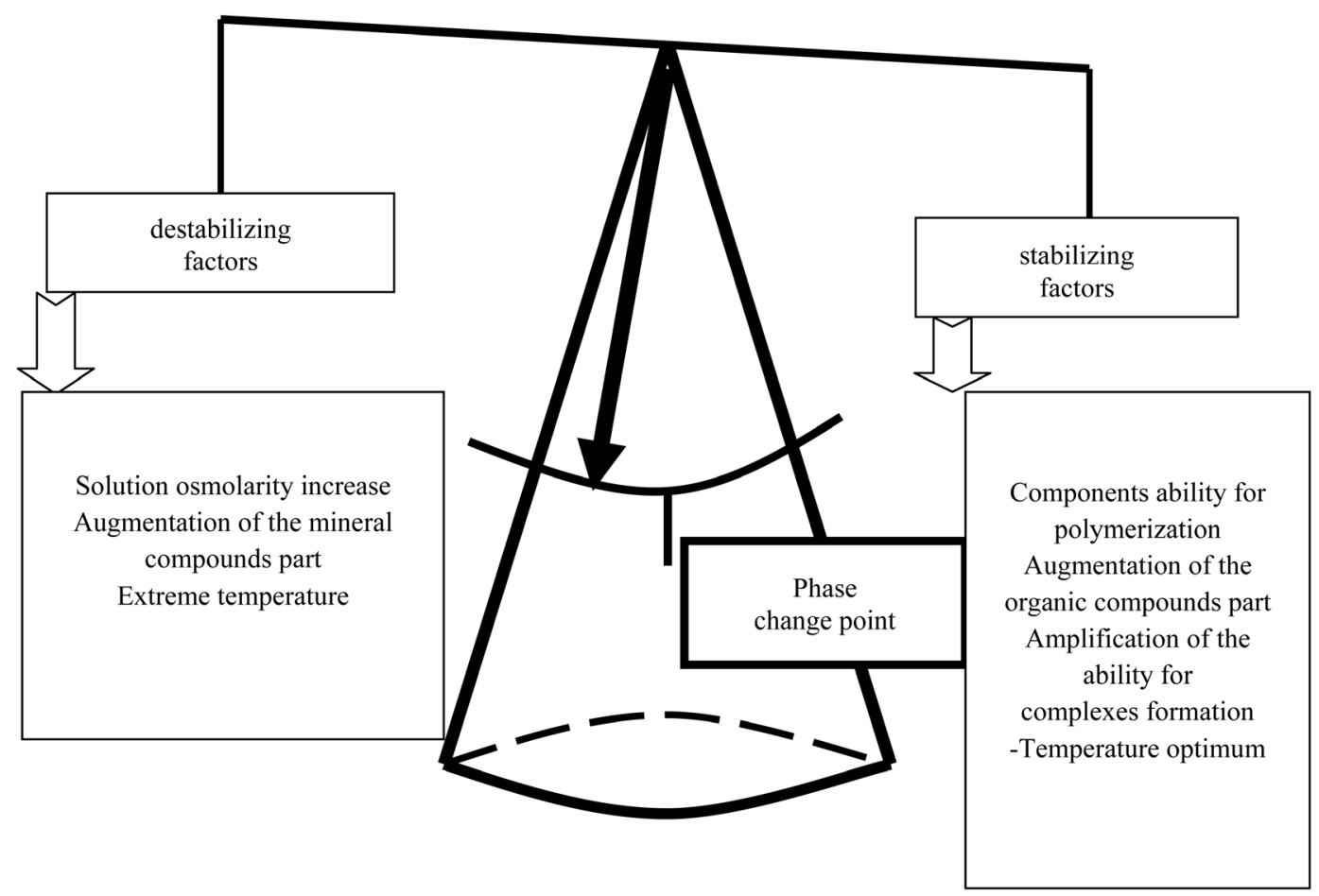

Figure 3. Conception of crystallogenic stability of the biofluid.

- yielding of crystal;

- pseudo-crystals forming;

- $\quad$ transfer to a liquid-crystalline state.

When the stabilizing factors predominate the drying leads to the total or partial crystallogenesis inhibition being realized by [13]:

- gel formation;

- sol formation.

We proposed a conception of the biological medium stability as a solution based on the application of such an experimental approach. The conception rested on the position that any input into a biosystem substance modulates the result of the starting biosubstratum dehydration and direct it to a liquid state conservation (stabilizing factor) or to a crystallization (destabilizing factor) taking into consideration the crystallogenesis macro- and micro-surroundings conditions [9] [21].

\section{Conclusions}

1) Metabolites were active modulatory factors of the biological fluids crystallogenesis. Introduced or fallen into a biological medium in vivo they took on properties of the stabilizing or destabilizing factors.

2) Injection of substrates of the lactate dehydrogenase (sodium lactate and sodium pyruvate) into the crystallizing specimen dose-dependently changed the result of dehydration of human urine.

3) Activation of the opposite enzyme reactions concerned with the substratum administration led to the similar antagonistic transformation of the biofluid crystallogenesis.

\section{References}

[1] Denisov, A.B. (2004) Algorithm for Evaluation of Crystal Figures Obtained after Drying of Mixed Saliva. Bulletin of Experimental Biology and Medicine, 138, 30-33. http://dx.doi.org/10.1007/BF02694466

[2] Savina, L.V. (1999) Crystalloscopic Structures of the Healthy and Sick Person Blood Serum. Sovetsakya Kuban, Krasnodar.

[3] Shabalin, V.N. and Shatokhina, S.N. (2001) Morphology of the Human Biological Fluids. Khrisopraz, Moscow. 
[4] Vorobiev, A.V., Vorobieva, V.A., Neshtakova, N.L., et al. (2002) Teziographic Diagnostics of Acute Lymphoblastic in Children. Nizhni Novgorod Medical Journal, 2, 61-64.

[5] Chuhman, T.P. (2000) Crystallographic Study of Dacryops at Inflammatory Eye Diseases. Ph.D. Dissertation, Samara State Medical University, Samara.

[6] Voltchetsky, A.L., Spasennikov, B.A., Agafonov, V.M., et al. (1999) Method Modification and Computerization of Teziographic Analysis. Human Ecology, 3, 38-42.

[7] Koledintsev, M.N. (1999) Clinical Properties of Dacryops Crystallographic Analysis at Some Eyes Pathology. Ph.D. Dissertations, Moscow State Stomatology University, Moscow.

[8] Tarusinov, G.A. (1994) Urine Crystallographic Study in Diagnostics of Diffuse Connective Tissue Diseases in Children. Pediatrics, 1, 55-57.

[9] Vorobiev, A.V., Martusevich, A.K. and Peretiagin, S.P. (2008) Biological Fluids and Substratum Crystallogenesis in the Organism's State Estimation. Nighny, Novgorod.

[10] Zubeeva, G.N., Motyleva, I.M., Potekhina, Yu.P., et al. (2001) Crystallographic Screening Method in Diagnostics and Treatment of Patients with Ciliary Arrhythmia. Clinical Laboratory Diagnostics, 9, 10.

[11] Dushkov, V.A., Kutnaya, G.B., Baibulatova, L.B., et al. (2005) Ozone Therapy Effectiveness Estimation at Patients with Chronic Cerebrovascular Insufficiency on Results of Blood Serum Morphological Analysis. Nizhni Novgorod Medical Journal, Appl. Ozone Therapy, 79-81.

[12] Kurnisheva, N.I., Nagornova, N.D., Deev, A.I., et al. (2003) Method of Involutory Ophthalmoendotoxicosis Estimation by Dacryops Fluorescence Study. Clinical Gerontology, 9, 91.

[13] Yakhno T.A. and Yakhno, V.G. (2007) Structural Evolution of Drying Drops of Biological Fluid. Technical Physics, 54, 1219-1227. http://dx.doi.org/10.1134/S1063784209080210

[14] Annarelli, C., et al. (2001) Crack Patterns in Drying Protein Solution Drops. European Physical Journal E, 5, $599-603$. http://dx.doi.org/10.1007/s101890170043

[15] Savina, L.V., Pavlishuk, S.A., Samsigin, V.Yu., et al. (2003) Polarization Microscopy in Methabolic Disorders Diagnostics. Clinical Laboratory Diagnostics, 3, 11-13.

[16] Zaleski, M.G. (2005) Dispersion of Mineral and Protein Components in Facia of Urine and "Lythos-System” Diagnosticum Mixture Drops. Herald of New Medical Technologies, 12, 93-94.

[17] Menshikov, V.V. (1988) Laboratory Tests in the Clinical Practice. Medicine, Moscow.

[18] Nazarenko, G.I. and Kishkun, A.A. (2000) Clinical Estimation of the Laboratory Analysis Results. Medicine, Moscow.

[19] Kamakin, N.F. and Martusevich, A.K. (2003) Modern Technologies in Crystalloscopic Identification of Human Organism Biological Fluids Composition. Human Ecology, 5, 23-25.

[20] Martusevich, A.K. (2006) Informational Physical and Biochemical Theory of Biocrystallization as Biological Fluids Morphology Reflection. Herald of Ural Medical Academic Science, 15, 112-113.

[21] Martusevich, A.K. and Kamakin, N.F. (2007) Crystallography of Biological Fluid as a Method for Evaluating Its Physicochemical Characteristics. Bulletin of Experimental Biology and Medicine, 143, 385-388.

http://dx.doi.org/10.1007/s10517-007-0118-7 